\title{
LA GUÍA DE EXPERTOS EN LAS UNIVERSIDADES PRIVADAS ESPAÑOLAS: ANÁLISIS DE SU PRESENCIA Y GESTIÓN PARA LA TRANFERENCIA DEL CONOCIMIENTO CIENTÍFICO
}

\section{THE EXPERT GUIDE IN SPANISH PRIVATE UNIVERSITIES: ANALYSIS OF THEIR PRESENCE AND MANAGEMENT FOR SCIENTIFIC KNOWLEDGE TRANSFER}

Aída María De Vicente Domínguez. Universidad de Málaga. España. aidamaria@uma.es

Nereida Cea Esteruela. Universidad de Málaga. España. nereidacea@uma.es

Mireya Rocio Carballeda Camacho. Universidad de Málaga. España. mcarballeda@uma.es

Cómo citar el artículo:

De Vicente Domínguez, A-M., Cea Esteruela, N., Carballeda Camacho, M-R. (2022). La guía de expertos en las universidades privadas españolas: análisis de su presencia y gestión para la tranferencia del conocimiento científico. Revista de Ciencias de la Comunicación e Información, 27, 61-75. http://doi.org/10.35742/rcci.2022.27.e247.

\section{RESUMEN}

La guía de expertos es un directorio que agrupa a los investigadores de una institución dispuestos a colaborar con los medios de comunicación y que permite a los periodistas localizarles de forma autónoma en un corto espacio temporal. Una herramienta comunicacional que permite optimizar la proyección mediática del capital intelectual de la organización, aumentando la visibilidad, marca y reputación de la institución. En este contexto, se investiga su presencia y gestión en las universidades privadas españolas. Los objetivos son averiguar su presencia o ausencia; identificar los motivos que han frenado su implementación digital; analizar su ubicación, tipo de formato e idiomas; investigar cómo se localiza a un experto/a; analizar los datos curriculares ofrecidos del investigador/a; desvelar qué datos curriculares ofrecen del experto/a; y dar a conocer las modalidades para contactar con el especialista. La metodología se basa en un análisis de contenido y el periodo de estudio es de febrero a junio de 2020. Los resultados indican una escasa presencia, si bien, siempre se ubican en las salas de prensa y su formato suele ser digital (no en pdf), permitiendo la búsqueda por nombre-apellidos o especialidad, principalmente sólo en español, ofreciendo poca información curricular de los investigadores y permitiendo contactar siempre a través del teléfono o e-mail institucional mediante un contacto tanto directo como mediado por el gabinete de comunicación. Se concluye con una visión global de su 
implementación y una identificación de las disfunciones y buenas praxis detectadas para transferir el conocimiento científico mediante esta herramienta organizacional.

PALABRAS CLAVE: comunicación organizacional; periodismo; guía de expertos; divulgación científica; universidad; gabinete de comunicación; Internet.

\section{ABSTRACT}

The guide of experts is a directory of researchers from an institution who are willing to collaborate with the media and allows journalists to locate them autonomously in a short period of time. It is a communication tool that optimises the media projection of the organisation's intellectual capital, increasing the visibility, branding and organisation's intellectual capital, increasing the visibility, brand and reputation of the institution. For this reason, its presence and management in Spanish private universities is investigated. The objectives are to ascertain their presence or absence from the guide; identify the reasons that have slowed down their digital implementation; analyse their location, type of format and languages; investigate how an expert is located; analyse the curricular data offered by the researcher; reveal the curricular data offered by the expert; and make known the methods for contacting the specialist. The methodology is based on a content analysis and the study period is from February to June 2020. The results indicate a poor presence, although they are always located in the press rooms and their format is usually digital (not in pdf), allowing the search by name-surname or speciality, mainly only in Spanish, offering little curricular information on the researchers and always allowing contact by telephone or institutional e-mail through both direct contact and contact mediated by the communication office. It concludes with an overview of its implementation and an identification of the dysfunctions and good practices detected for transferring scientific knowledge through this organisational tool.

KEYWORDS: organisational communication; journalism; expert guide; scientific dissemination; university; communication office; Internet.

\section{INTRODUCCIÓN}

Esta investigación analiza la presencia y gestión o implementación, de una herramienta de relación con los medios, en los gabinetes de comunicación de las universidades privadas españolas. Se trata de la guía de expertos: un directorio conformado por los especialistas de la institución, dispuestos a colaborar con los profesionales de la información. Un servicio que permite a los periodistas localizarlos, de forma autónoma en el área específica que precisen, y establecer contacto directo con el docente investigador en un corto espacio de tiempo.

Un recurso que se considera significativo analizar porque la relación con los medios es un área clave en todo gabinete de comunicación. Y la guía de expertos, a los gabinetes de comunicación universitarios, le permite gestionar la principal demanda solicitada por los periodistas: localizar y establecer contacto con los profesores e investigadores universitarios. Y es que como desvelan Repiso Y Chaparro (2018) son "los principales responsables de la actividad universitaria de la se hacen eco los 


\section{LA GUÍA DE EXPERTOS EN LAS UNIVERSIDADES PRIVADAS ESPAÑOLAS: ANÁLISIS DE SU PRESENCIA Y GESTIÓN PARA LA TRANFERENCIA DEL CONOCIMIENTO CIENTÍFICO}

medios" (p. 87). Es así, que esta herramienta puede ayudar a este sector a optimizar diversos objetivos que deben cumplir las universidades como el indicado por Rowe (2005): "situar el conocimiento académico que generan en el discurso diario de los medios de comunicación" (citado en Repiso, Merino-Arribas y Chaparro-Domínguez, 2016); o el expuesto por Lascuarin y Sanz (2009) "que los científicos faciliten, transfieran y divulguen sus conocimientos" (p. 11). Por ello, Parejo (2015) considera esta herramienta de suma utilidad pues aporta transferencia y revaloriza la imagen de la institución, motivos por los que advierte como "tendría que convertirse en prioridad para las universidades a la hora de trazar sus planes estratégicos" (p. 534).

En concreto, este estudio analiza su presencia y gestión en el entorno digital. Estudios precedentes han abordado cómo los gabinetes de comunicación universitarios han adoptado las clásicas herramientas de relación con los medios al soporte digital (notas de prensa o ruedas de prensa, entre tantas otras). O cómo han implementación las emergentes (redes sociales, podcasts, blogs, radios y televisiones universitarias, etc...). Tómese como referencia algunos de los estudios más recientes: Cestino (2020); García-García (2018); Martín-González y Santamaría (2017); Pham et al. (2017); Simón (2016); Shields (2016); Valerio et al. (2015). Sin embargo, la adaptación on line para gestionar la demanda de su capital intelectual con los media, como la guía de expertos, apenas ha sido analizada.

Las primeras investigaciones sobre la guía de expertos en la universidad española las realizaron Palomares et al., (2003) que aportaron una propuesta para crear una guía de expertos universitaria para traductores. Y Palomares y Accino (2003) que describieron la estructura seguida para crear una guía de expertos para la Traducción (GET). Será en 2005 cuando Rodríguez-Wangümert y González-Afonso desvelen datos pioneros de la implantación de una guía de expertos impresa, destinada a los medios informativos, en un gabinete de comunicación universitario español: la Universidad de La Laguna. Después, Carrillo y Parejo (2009) analizan su presencia en las salas de prensa virtuales de las universidades públicas españolas.

Una variable también abordada recientemente por Romo, Espinosa y Gómez (2020), cercando la muestra a las diez primeras universidades españolas del ranking de Scimago. Si bien, López-Hernández y Domínguez-Delgado (2018) describen como se gestionó un directorio web de fuentes expertas de la Universidad de Sevilla: pero esta web no es gestionada por un gabinete de comunicación, sino fruto de un proyecto de innovación docente cuya base de datos no está destinada a los media, sino en exclusiva al alumnado de la Universidad de Sevilla para acceder a fuentes fiables para sus trabajos de investigación.

No hay datos, hasta el momento, de su implementación en la universidad privada española. Tan sólo, un caso de estudio específico centrado en la Universidad de Navarra (De Vicente y Sierra, 2020), y los aportados colateralmente por Parejo (2015) pues su estudio no se centra en estas guías sino en el nivel de adecuación de las salas de prensa en función de las herramientas que usa.

Ahora se pretende aportar los primeros datos sobre cómo han creado las universidades privadas españoles esta herramienta, que es considera por un panel de 27 expertos conformado por "responsables y técnicos de gabinetes de comunicación de universidades públicas y privadas, directores de unidades de cultura científica y 
asociaciones de comunicación, así como periodistas en ejercicio en medios de comunicación del panorama nacional" (p.31), una de las principales herramientas que debe tener todo gabinete de comunicación universitario.

Datos de utilidad para aquellas instituciones que quieran crear una guía de expertos pues "aprender a gestionar el capital intelectual de las organizaciones se revela como una de las tareas fundamentales que se están implementando en todas las organizaciones competitivas" (Palomares et al. 2003: 234). Y en el caso de las universidades privadas, el incremento de su posicionamiento es muy necesario, pues se trata de organizaciones que se mantienen de fondos propios.

\section{OBJETIVOS}

En este contexto, el objetivo principal de la comunicación es investigar las guías de expertos en el soporte digital en las universidades privadas españolas. Y los objetivos específicos son:

- Computar la presencia y ausencia de la guía de expertos en las universidades privadas españolas.

- Identificar los motivos que han frenado la adaptación de este servicio al entorno digital.

- Analizar su ubicación y tipo de formato.

- Averiguar en cuantos idiomas se puede consultar.

- Investigar cómo es el proceso de consulta para localizar a un experto/a.

- Identificar los datos curriculares que se ofrecen del personal docente investigador.

- Desvelar qué datos ofrecen para establecer contacto con el experto/a seleccionado.

\section{METODOLOGÍA}

La metodología empleada se ha basado en las siguientes fases:

- Revisión bibliográfica en bases de datos nacionales e internacionales para comprobar la viabilidad del tema objeto de estudio, así como, las variables o enfoques previamente analizados.

- La selección de la muestra se obtiene del listado de universidades privadas españolas aportadas por el Registro de Universidades, Centros y Títulos Propios (RUCT) del Ministerio de Ciencia, Innovación y Universidades del Gobierno de España. Para el estudio se seleccionan todas las universidades 
excepto aquellas que cumplen los siguientes criterios de exclusión: no tener profesorado y, por tanto, es lógico la ausencia de este recurso.; no existen, aunque estén registradas; o son un centro adscrito a una universidad española. Se analizan durante el periodo comprendido de febrero a junio de 2020.

Tabla 1. Universidades privadas españolas /año 2020

\begin{tabular}{|l|l|l|}
\hline $\begin{array}{l}\text { Universidad Alfonso X el } \\
\text { Sabio }\end{array}$ & Universidad de Navarra & $\begin{array}{l}\text { Universidad Internacional } \\
\text { de Catalunya }\end{array}$ \\
\hline $\begin{array}{l}\text { Universidad Camilo José } \\
\text { Cela }\end{array}$ & $\begin{array}{l}\text { Universidad Vic-Universidad } \\
\text { Central de Catalunya }\end{array}$ & $\begin{array}{l}\text { Universidad Pontifica de } \\
\text { Salamanca }\end{array}$ \\
\hline Universidad de Deusto & $\begin{array}{l}\text { Universidad Católica Santa } \\
\text { Teresa de Jesús de Ávila }\end{array}$ & Universidad Ramón Llull \\
\hline $\begin{array}{l}\text { Universidad del Atlántico } \\
\text { medio }\end{array}$ & $\begin{array}{l}\text { Universidad Cardenal Herrera } \\
\text { CEU }\end{array}$ & $\begin{array}{l}\text { Universidad Europea } \\
\text { Miguel de Cervantes }\end{array}$ \\
\hline $\begin{array}{l}\text { Universidad Europea de } \\
\text { Canarias }\end{array}$ & Universitat Abat Oliva Ceu & $\begin{array}{l}\text { Universidad Francisco de } \\
\text { Vitoria }\end{array}$ \\
\hline $\begin{array}{l}\text { Universidad Europea de } \\
\text { Madrid }\end{array}$ & Universidad San Pablo CEU & $\begin{array}{l}\text { Universidad Fernando- } \\
\text { Pessoa-Canarias }\end{array}$ \\
\hline $\begin{array}{l}\text { Universidad Europea de } \\
\text { Valencia }\end{array}$ & Universidad Católica de & $\begin{array}{l}\text { Universidad Antonio de } \\
\text { Nebrija }\end{array}$ \\
\hline $\begin{array}{l}\text { Universidad Europea del } \\
\text { Atlántico }\end{array}$ & Universidad Internacional de & $\begin{array}{l}\text { Universidad a Distancia } \\
\text { de Madrid }\end{array}$ \\
\hline $\begin{array}{l}\text { Universidad Católica de San } \\
\text { Antonio }\end{array}$ & Universidad de San Jorge & Mondragón Universitatea \\
\hline $\begin{array}{l}\text { Universidad Pontifica de } \\
\text { Comillas }\end{array}$ & Universidad Loyola de & IE Universidad \\
\hline $\begin{array}{l}\text { Universidad Oberta de } \\
\text { Catalunya }\end{array}$ & Universidad Isabel I de Castilla & $\begin{array}{l}\text { Universidad Internacional } \\
\text { de la Rioja. }\end{array}$ \\
\hline
\end{tabular}

Fuente: Registro de Universidades, Centros y Títulos Propios (RUCT)

1. La metodología empleada para investigar la presencia de la guía de expertos es un sistema binomial (Si/No). Se localiza exponiendo "guía de expertos" en el buscador principal de la web de la institución y en el buscador de la sala de prensa virtual. En caso negativo, se establece contacto telefónico con el gabinete para confirmar su ausencia pues pudiera tener otra denominación, o bien, que los buscadores no recuperaran este servicio.

2. Para conocer los motivos que expliquen la ausencia de este recurso de relaciones públicas se procede a enviarles esta cuestión vía correo electrónico, de contestación abierta, hasta en tres ocasiones. Un último intento se establece por vía telefónica. El nivel de confianza de los casos analizados es del 95\% y un margen de error inferior al $10 \%$.

Para analizar la ubicación, formato, idioma y sistema de localización se toma como referencia la metodología usada por Parejo (2015) en su tesis doctoral, conformada por 3 niveles de adecuación: alto (1), medio (2) y bajo (3) expuesta en la tabla 1. Así, el código número numérico creado para analizar este nivel en la adecuación en la ubicación es: alto (1) si está en la sala de prensa; medio (2) si está ubicado en un vicerrectorado; y bajo (3) en caso de estar ubicado en otras secciones. Para el formato se otorga un nivel alto (1) si es digital, un nivel medio (2) si es un volcado en pdf y un nivel bajo (3) si es volcado es desde excel. Si bien, para analizar el nivel de adecuación 
del número de idiomas en el que se pueden realizar las búsquedas, se otorga nivel alto (1) a las que permiten localizar a un experto/a en más de 3 idiomas; nivel medio (2) a poder buscarlos en más de dos idiomas; y nivel bajo (3) si permite localizar al experto/a en sólo un idioma.

Asimismo, los códigos usados para investigar el nivel de adecuación según el sistema de localización han sido otorgando el nivel alto (1) a las guías digitales que permiten localizarles por nombre o apellidos y especialidad; un nivel medio (2) a las que permiten su localización sólo por especialidad y un nivel bajo (3) para las búsquedas que sólo son posibles mediante el nombre y el apellido.

Tabla 2: Metodología para analizar el nivel de adecuación de las variables ubicación, formato, traducción y sistema de consulta.

\begin{tabular}{|l|l|l|l|l|}
\hline $\begin{array}{l}\text { Nivel de } \\
\text { ubicación }\end{array}$ & Ubicación & Formato & Traducción & Sistema de consulta \\
\hline Alto & 1.Sala de prensa & 1. Digital & $\begin{array}{l}\text { 1. Tres o más } \\
\text { idiomas }\end{array}$ & $\begin{array}{l}\text { 1. Nombre, apellido y } \\
\text { especialidad }\end{array}$ \\
\hline Medio & 2.Vicerrectorado & 2. pdf & 2. dos idiomas & 2. Especialidad \\
\hline Bajo & 3.Otras secciones & $\begin{array}{l}\text { 3. Tablas } \\
\text { excell }\end{array}$ & 1. Un idioma & 3. Nombre y apellido \\
\hline
\end{tabular}

Fuente: elaboración propia basado en la metodología de Parejo (2015)

- Para conocer los contenidos aportados por el experto se elabora un doble análisis. El primero, destinado a investigar si se aporta el currículo investigador del experto mediante un sistema binomial (si/no). Y un segundo, para conocer el tipo de currículum que se ofrece analizándose mediante dos opciones: currículo personalizado (aquel que está escrito por el investigador) o currículo indexado (aportando el acceso a las bases de datos que recogen y tienen actualizada su producción científica como Dialnet, Google Scholar, Orcid, etc). Otorgando el valor numérico 1 al currículo personalizado y un 2 al currículo indexado. Así como, para analizar si aportan la actividad de transferencia del experto/a se opta por un sistema binomial (si/no).

- La metodología para analizar el sistema de recuperación o contacto final con el experto se estructura creando dos categorías: acceso directo (aquel que permite al periodista localizar personalmente al experto) o acceso mediado (aquel que precisa contactar con el gabinete de comunicación universitario para que gestione el contacto). Se otorga un 1 al acceso directo y un 2 al acceso mediado.

- Se establecen, asimismo, dos opciones para analizar el tipo de acceso directo: contacto institucional (aportar el teléfono o email institucional de experto), o contacto específico (dar el contacto directo con la fuente como puede ser a través de un móvil). Otorgando un 1 al contacto institucional y un 2 al contacto específico. Y para analizar las modalidades de acceso mediado se establecen los siguientes códigos: formulario (necesidad de rellenar los datos solicitados a través de la plataforma) o telefónico (se aporta el contacto del responsable de 
LA GUÍA DE EXPERTOS EN LAS UNIVERSIDADES PRIVADAS ESPAÑOLAS: ANÁLISIS DE SU PRESENCIA Y GESTIÓN PARA LA TRANFERENCIA DEL CONOCIMIENTO CIENTÍFICO

comunicación de la institución), otorgando el valor numérico de 1 al formulario y de 2 al telefónico.

\section{RESULTADOS Y DISCUSIÓN}

\subsection{Presencia y ausencia de la guía de expertos}

Los datos resultantes de los primeros objetivos, computar su presencia y los motivos de su ausencia, desvelan que tan sólo un $33,33 \%$ de las universidades privadas españolas disponen de una guía de expertos en 2020, lo que indica que está poco implantada en las universidades privadas, pues un $66,67 \%$ no disponen de este servicio.

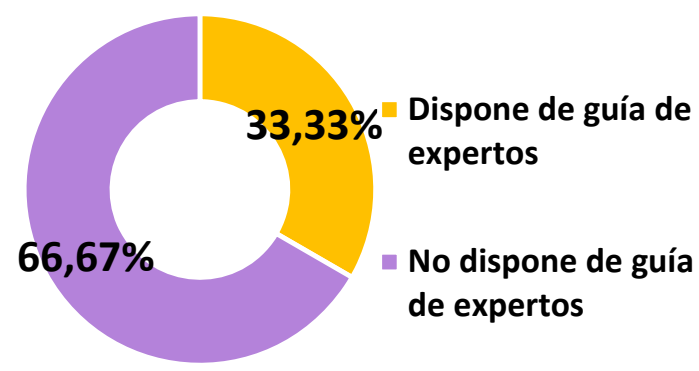

Gráfico 1. Presencia de la guía de expertos en las universidades privadas españolas

Fuente: Elaboración propia

Unos resultados que coinciden con los aportados por Parejo (2015): "se trata de una herramienta poco extendida entre las universidades" (p. 479) a pesar de que para los expertos su inclusión en las salas de prensa virtuales sea muy bien valorada. No obstante, se ha producido un leve incremento de su presencia, pues Parejo (2015)

indicaba que solo estaba en "un escaso $20 \%$ " en todas las universidades españolas" (p. 575) y los datos obtenidos en 2020 indican que se eleva al 33,33\%. Asimismo, en el momento del estudio se destaca que la Universidad a Distancia de Madrid (UDIMA) si dispone de una guía impresa, enviada a los medios, que está pendiente de subirse a la web, lo que incrementará estos datos.

Los motivos que explican la ausencia de este recurso en las universidades privadas españolas $(66,67 \%)$ son: desconocimiento de esta herramienta de relaciones públicas; los constantes cambios en la plantilla de los profesores o las circunstancias laborales de los docentes; dudas sobre la protección de datos; disponer de una guía o documento interno; satisfacción con el método usado para gestionar las demandas de los periodistas; para tener un mayor control de la relación de los expertos con los 
media; considerar que así aportan el experto más adecuado o que ganan tanto en eficiencia como en agilidad de gestión; porque a los medios les gusta llamar al gabinete; que es mejor trabajar bajo demanda; imposibilidad de actualizarla; resultarles más fácil su gestión; no tener el respaldo de la institución; que las circunstancias han obligado a priorizar otros asuntos; no tener necesidad porque es una universidad pequeña y se conocen todos; estar en proceso o en estudio; no haber unos motivos específicos; o no saberlo.

Y se destaca que el sistema que usan las universidades que no han implementado la guía de expertos para atender las demandas de los medios es el clásico sistema expuesto por Abramczyk (1990), quién hace décadas incidía en la necesidad de unos asesores de prensa en los centros de investigación para facilitar el contacto (citado en Moreno, 2004, p.160). Pero destacamos otras prácticas significativas que se usan para aumentar la visibilidad de la institución usando los soportes digitales:

- Enviar a los medios un boletín con propuestas de actualidad junto a sus mejores expertos en dicha especialidad.

- Mandar un documento identificando las especialidades que se investigan en la universidad (sin identificarlos con expertos específicos).

- Remitirles un listado con los expertos considerados más significativos por el gabinete de la institución.

\subsection{Ubicación, formato y número de idiomas}

Los resultados obtenidos sobre la ubicación de la guía de expertos en la web universitaria, desvelan que en el $100 \%$ de la muestra están en las salas de prensa obteniendo un nivel de adecuación alto. En el estudio precedente de Parejo (2015) desvelada que alguna de las universidades españolas la tenía ubicada en el vicerrectorado de investigación. Esto implica una unificación en el criterio para ubicar este recurso.

El formato usado, según los datos obtenidos, tiene un nivel de adecuación alto en el $90,9 \%$ de la muestra porque han creado su sistema de software, con su respectivo almacenamiento de datos al que se accede indexando las palabras clave en los buscadores de localización. Sólo un 9,09\% presenta un nivel de adecuación bajo, al aportar un volcado en Excel con los expertos/as que quieren colaborar con los medios de comunicación, lo que respondería al denominado "modelo facsimilar", concepto acuñado por Cabrera (2001). Un modelo caracterizado por adaptarse de manera sencilla y poco elaborada a las características propias del medio on line junto a la simplicidad del diseño.

Los datos sobre el nivel de adecuación según el número de idiomas para consultar el contenido de la guía de expertos, desvelan que en un $81,81 \%$ es un nivel bajo (sólo puede usarse en español) frente a un $18,18 \%$ que tiene un nivel alto pues permite localizar a los expertos/as en tres idiomas (español, inglés y el autóctono de esa área geográfica). Este nivel bajo coincide con el obtenido por Parejo (2015), puesto que el $96 \%$ de todas las universidades españolas que tenían guía de expertos se ofrecía en un idioma. Por tanto, en general, no se aprovecha este recurso para tener una mayor 
proyección en la prensa extranjera. No obstante, se destaca que un sólo idioma también puede aumentar la internacionalización en áreas geográficas que emiten programas en español. Tómese como referencia la guía de expertos de la Universidad Navarra que consiguió servir como fuente a un programa para españoles emitido en Baviera (Alemania).

Formato

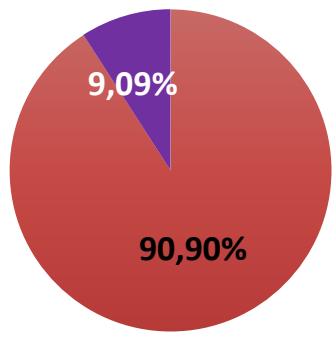

Digital $\quad$ Excel
Idioma

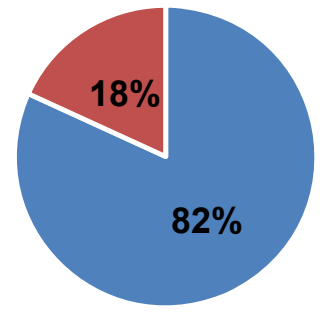

- Un idioma - Tres idiomas

Gráfico 2. Formato y número de idiomas para consultar la Guía de expertos

Fuente: elaboración propia

\subsection{Localización, contenido y método para contactar con el experto}

El sistema de localización de los expertos presenta un nivel de adecuación alto en el $81,81 \%$ de la muestra puesto que permite la búsqueda tanto por especialidad como por nombre y apellido del experto/a; un $9,09 \%$ posee un nivel de adecuación medio, porque sólo permite la búsqueda por especialidad; y otro $9,09 \%$ tiene un nivel bajo al cercarla solo por nombre y apellidos, lo que implicaría que el profesional tuviese identificado al experto previamente, cuando lo usual es que se localice por temática ante el desconocimiento de especialistas en un determinado campo. Y se destaca que todas las universidades especifican en su buscador cómo realizar la búsqueda, menos un $9,09 \%$ que corresponde a la implantación de un volcado de los datos en Excel.

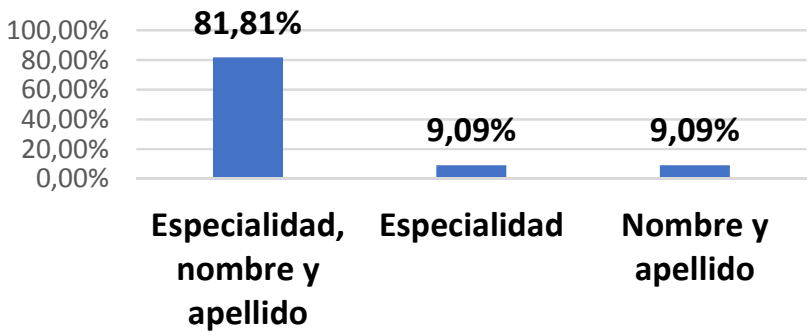

Gráfico 3. Sistema de localización de la guía de expertos

Fuente: elaboración propia 
Los contenidos referidos al currículum investigador indican que tan sólo un $45,45 \%$ de las universidades objeto de estudio aportan estos datos, frente a un $54,54 \%$ que no ofrecen datos sobre su actividad investigadora. Y del $45,45 \%$ que lo exponen, sólo un $15,15 \%$ exhibe un currículo indexado a través de sus grupos de investigación o diversas bases de datos como Google scholar, Scopus, Orcid, o Publons, entre otras. Asimismo, también se caracteriza por no insertar su actividad de divulgación científica: tan sólo un $27,27 \%$ lo muestra, frente al $72,72 \%$ que carecen de esta información.

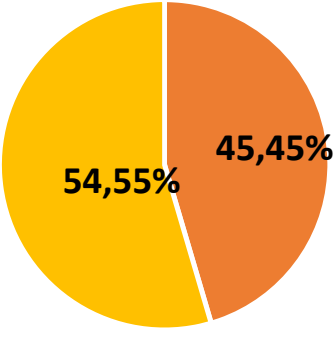

Incluyen datos de actividad

investigadora

No incluyen datos de actividad investigadora

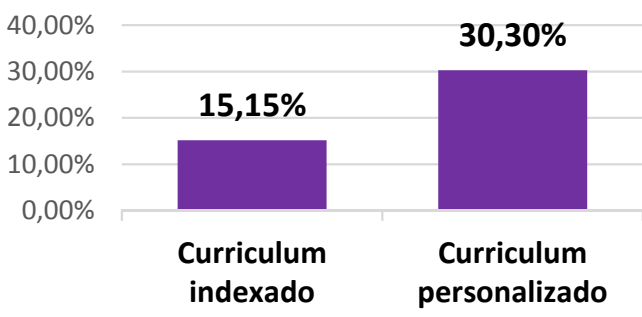

Datos sobre actividad investigadora...

Gráfico 4. Datos ofrecidos de la actividad investigadora del experto/a.

Fuente: elaboración propia

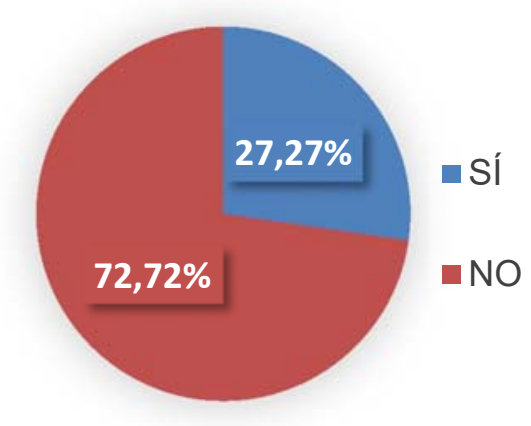

Gráfico 5. Datos ofrecidos de la transferencia científica del investigador/a

Fuente: elaboración propia

Y los resultados indican que un $36,36 \%$ de la muestra objeto de estudio ofrece un acceso directo, permitiendo que sea el periodista quien realice personalmente el contacto con el experto. Si bien, en el $100 \%$ de los casos se ofrece un contacto institucional (número de teléfono y e-mail institucional del experto/a) y en un $20,77 \%$ lo combina con un acceso específico (aportando el número móvil del experto/a), lo que permite su localización en cualquier franja horaria.

Y el 63,63 \% de la muestra opta por un acceso mediado siendo necesario establecer contacto con el gabinete de comunicación. Y los resultados desvelan que un 18,18\% lo hacen mediante un formulario: indica al periodista que rellene un formulario 
(nombre, medio, teléfono y e-mail obligatorio, y unas observaciones opcionales) o bien (nombre, apellidos, medio para el que trabaja, teléfono de contacto, e-mail, materia y descripción de la consulta); y un $45,45 \%$ solicita al periodista que establezca contacto con el gabinete mediante un número telefónico: si bien tan solo en el $27,27 \%$ el responsable del gabinete de comunicación aporta junto al e-mail y teléfono institucional un teléfono móvil $(18,18 \%)$.
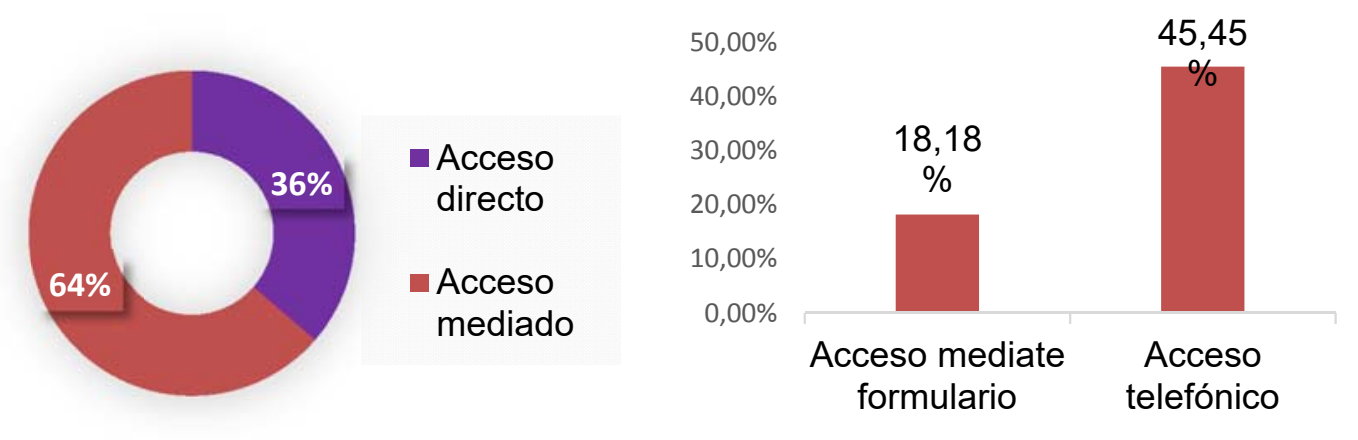

Gráfico 6. Modalidad de acceso al experto/a

Fuente: elaboración propia

\section{CONCLUSIONES}

El objetivo principal de la investigación ha sido averiguar cómo las universidades privadas españolas han implementado, la guía de expertos, en el soporte digital. Y de la visión global obtenida concluimos:

- Aunque los expertos consideran la guía de expertos como una de las principales herramientas que deben formar parte de los gabinetes de comunicación universitarios, más de la mitad de las universidades privadas españolas carecen de este servicio digital. No obstante, algunos de los motivos que explican su ausencia son: estar en proceso, estar pendiente de subirse a la red, o ser un proyecto futuro. Causas que hacen prever un incremento en un futuro, en el sector analizado, aunque no en su totalidad. No obstante, muestra como la universidad sigue invirtiendo para lograr una mayor proyección de la institución en el entorno digital.

- El desconocimiento de esta herramienta es otro de los motivos que frenan la gestión del conocimiento tácito, considerado como uno de los grandes retos de toda organización competitiva. Esta causa junto a las relacionadas con una proyección futura de su implementación otorga significatividad a la necesidad de ofrecer pautas de referencias, como las expuestas en esta investigación, para gestionar una guía de expertos en el soporte digital, y optimizar las actuales.

- Una gestión que consiste en atender los intereses del público externo al que va 
dirigido, los periodistas, y que en su contexto global está conformada por buenas praxis, pero también con deficiencias que obstaculizan una mejor proyección de los activos estratégicos del conocimiento para incrementar la visibilidad, el valor y la marca de la institución universitaria en los medios de comunicación.

- La adaptación inicial del sistema de búsqueda y acceso, es decir, cómo buscaría el periodista esa herramienta y accedería fácilmente al recurso, es atendido correctamente por todas las universidades. Todas la ubican en la sala de prensa: lugar al que accederían porque es donde se gestiona la relación con los medios.

- En una segunda fase, localizado el recurso de este servicio, el periodista precisa un proceso de consulta para realizar la búsqueda y que este sea fácil. Proceso que está prácticamente bien adaptado en casi todas las universidades porque explican al usuario qué palabras clave deben usar para realizar la búsqueda. Si bien, aunque la mayoría permiten localizar al experto/ a por nombre apellidos y especialidad; se detecta en algunas la deficiencia como permitir sólo localizarlos a través de su nombre o apellidos, lo que obliga al periodista a conocer al experto previamente. $Y$ es que lo usual es no conocer las identidades de los experto/as, sino localizarlos por temáticas.

- Los contenidos recuperados por el experto también presentan deficiencias. Se tiende a no identificar los datos curriculares y tampoco la actividad divulgadora del experto/a. Lo que obstaculizada otra de las necesidades de los periodistas para calibrar el grado de especialización de la fuente, o realizar una comparativa entre diversas fuentes para optar por una u otra en función del tema y enfoque de la pieza informativa. Una deficiencia cuya implementación ayudaría a superar otro de los problemas que obstaculizan su implementación: la necesidad de estar actualizando los datos. Y es que mediante currículo indexado sus publicaciones estarían siempre actualizadas.

- Estudios futuros analizarán la presencia de la guía de expertos en las universidades públicas españolas; investigarán el nivel de adecuación de esta herramienta en el cómputo total de universidades en España.; y analizarán la presencia de las universidades que tienen guías de expertos en los medios de comunicación.

\section{REFERENCIAS}

Abramczyk, J. (1990). La universidad y el periodismo científico. Arbor, ciencia, pensamiento y cultura, (534), 157-162.

Cabrera, MA. (2001). Convivencia de la prensa escrita y la prensa on line en su transición hacia el modelo de comunicación multimedia. Estudios sobre el Mensaje Periodístico, (7), 71-78. 
LA GUÍA DE EXPERTOS EN LAS UNIVERSIDADES PRIVADAS ESPAÑOLAS: ANÁLISIS DE SU PRESENCIA Y GESTIÓN PARA LA TRANFERENCIA DEL CONOCIMIENTO CIENTÍFICO

Calvo-Roy, A. (2015). Comunicación de la ciencia hoy: una visión personal y un decálogo añadido. Panace@: Revista de Medicina, Lenguaje y Traducción, 16 (42), 134-14.

Carrillo, MV., Castillo, A y Parejo, M. (2017). La gestión de la información universitaria a través de las salas de prensa alojadas en sus sedes web: propuesta de modelo para su gestión. Informação \& Sociedade, 27(1).

Carrillo, MV y Parejo, M. (2009). Las salas de prensa virtuales de los gabinetes de comunicación de las universidades públicas españolas. En: IV Congreso de la CiberSociedad. Crisis analógica, futuro digital.

Cestino-González, E. (2020). Aproximación al estudio de la estrategia de comunicación de las universidades andaluzas en Linkedln. Ámbitos. Revista Internacional de Comunicación, 171-187. https://doi.org./10.12795/Ambitos.2020.i48.09

Chapleo, C. (2010). What defines "successful" university brands? International Journal of Public Sector Management, n. 23, 2, 169-183. https://doi.org/10.1108/09513551011022519/

Codina, L. (2000). Parámetros e indicadores de calidad para la evaluación de recursos digitales. En: La gestión del conocimiento: retos y soluciones de los profesionales de la información: VII Jornadas Españolas de Documentación, 135-144.

Cortassa, C. (2012). La ciencia ante el público. Dimensiones epitémicas y culturales de la comprensión pública de la ciencia. Eudeba.

García-García, M. (2018). Universidad y medios sociales. Gestión de la comunicación en la universidad española" Revista Prisma Social, 22, 20-36.

Gómez-Camarero, C., Rodríguez-Reina, MP., Faber-Benítez, P., Pinto-Molina, M., Palomares Perraut, R., Corpas Pastor, G., Amaya-Galván, MC. y AccinoDomínguez, JA. (2003). La guía de expertos: una propuesta para la creación de una comunidad virtual universitaria de conocimiento para la traducción. En: Los sistemas de información en las organizaciones: eficacia y transparencia, $8^{\circ}$ Jornadas Españolas de Documentación, 231-239.

López Hernández, MA y Domínguez, R. (2017). Directorio Web de fuentes de información expertas para estudiantes de periodismo. En: JL. González-Esteban y García-Avilés, JA. (coords.), Mediamorfosis. Radiografía de la innovación en periodismo (237-254).

Lazcuarin, ML. y Sanz, E. (2009). La divulgación científica en el entorno universitario. En M. Borges(coord.) A ciencia da informacao criadora do cohecimiento (507515).

Martín-González, Y. y Santamaría, R. (2017). Universidades españolas en YouTube: gestión de canales institucionales y de sus contenidos. Cuadernos de documentación multimedia, https://doi.org/10.5209/CDMU.57970 
Moreno, C. (2004). La información científico-técnica. En: J.Fernández del Moral(coords). Periodismo especializado, 239-260.

Palomares-Perraut, R. y Accino-Domínguez, JA. (2003). El diseño de un Sistema de Información on line para la traducción profesional: La Guía de Expertos para la Traducción (GET). En: Actas del I Congreso Internacional de la Asociación Ibérica de Estudios de Traducción e Interpretación, 671-679.

Parejo-Cuellar, M. (2015). Los gabinetes de comunicación de las universidades españolas: propuesta de modelo y análisis de las salas de prensa universitarias. Tesis doctoral.

Pham, Hiep-Hung, Farrell, K., Vu, Huyen-Minh, Vuong, Quan-Hoang, y Napier, N. (2017). Using YouTube video to promote university: a content analysis. Technics Technologies Education Management (TTEM), 12(1) 58-72. Recuperado de https://papers.ssrn.com/sol3/papers.cfm?abstract id=2959229

Repiso, R., Merino-Arribas, A., Chaparro-Domínguez, MA. (2016). Agrupación de las universidades españolas en la prensa impresa nacional. Revista española de documentación científica, 39(2) 131. http://dx.doi.org/10.3989/redc.2016.2.1292

Repiso, R., Chaparro-Domínguez, MA. (2018). Universidades españolas en la prensa extranjera. Análisis de su cobertura periodística. El profesional de la información, 27(1) 86-94. https://doi.org/10.3145/epi.2018.ene.08

Rodríguez-Wangüemert, C., González-Afonso, C. (2005). La Comunicación de la Universidad: Guía de Expertos de La Laguna. En: MI. De Salas Nestares, AB. Fernández-Souto y F. Martínez-Vallvey. La Universidad en la comunicación, la comunicación en la Universidad. Edipo.

Romo, LM., Espinosa, ME. y Gómez Crisóstomo, R. (2020). Utilización de la Guía de expertos en las universidades españolas. En: $V$ Congreso de Comunicación y pensamiento, https://comunicacionypensamiento.org/inicio/ponencia/utilizacionde-las-guias-de-expertos-en-las-universidades-espanolas/

Rowe, D. (2005). Working Knowledge Encounters: Academics, Journalists and the Conditions of Cultural Labour. Social Semiotics, 15(3), 269-288. http://dx.doi.org/10.1080/10350330500310046.

Shields, R. (2016). Following the leader? Network models of 'world-class' universities on Twitter. International Journal of Educational Technology in Higher Education, v. 12, 50-63. https://link.springer.com/article/10.1007/s10734-015-9900-z

Simón, JE. (2016). Uso de las redes sociales por las universidades públicas andaluzas. Análisis de contenido de la cuenta oficial Twitter. Estudios del mensaje periodístico, 23(1), 631-645. https://doi.org/10.5209/ESMP.55618/

\section{AUTORES:}

\section{Aida María de Vicente Domínguez}


LA GUÍA DE EXPERTOS EN LAS UNIVERSIDADES PRIVADAS ESPAÑOLAS: ANÁLISIS DE SU PRESENCIA Y GESTIÓN PARA LA TRANFERENCIA DEL CONOCIMIENTO CIENTÍFICO

Doctora en Comunicación por la Universidad de Granada. Licenciada en Periodismo por la Universidad Carlos III de Madrid y Licenciada en Humanidades por la Universidad de Navarra. Especializada (máster) en dirección de comunicación y periodismo científico, sus principales líneas de investigación.

Orcid: https://orcid.org/0000-0002-8085-5097

Google scholar: https://scholar.google.es/citations?user=Uh-

SVXEAAAAJ\&hl=es\&oi=sra

Dialnet: https://dialnet.unirioja.es/servlet/autor?codigo=2915954

\section{Nereida Cea Esteruelas}

Doctora en Ciencias de la Información en la Universidad de Navarra. Entre sus líneas de investigación destaca el estudio de los mercados de la comunicación y la economía de los medios, con especial atención a los medios digitales. En el ámbito profesional ha trabajado en La Verdad (Grupo Vocento), La Economía (Grupo Intereconomía), El Mundo (Unidad Editorial). En el área de la comunicación política einstitucional, fue responsable de comunicación de la Cámara de Comercio de Murcia y asesora en la Consejería de Presidencia de la Comunidad Autónoma de la Región de Murcia.

Orcid: https://orcid.org/0000-0002-7733-9366, Dialnet:

https://dialnet.unirioja.es/servlet/autor?codigo $=1978623$

\section{Mireya Rocio Carballeda Camacho}

Licenciada en Comunicación Audiovisual por la Universidad de Málaga. Doctoranda en el Programa Interuniversitario de Comunicación.

Orcid: https://orcid.org/0000-0002-5269-2489

Google scholar: https://scholar.google.es/citations?user=nJ-

tb4wAAAAJ\&hl=es\&oi=sra 